\title{
Effect of a Mass Casualty Incident: Clinical Outcomes and Hospital Charges for Casualty Patients Versus Concurrent Inpatients
}

\author{
Mahshid Abir, MD, MSc, Hwajung Choi, PhD, Colin R. Cooke, MD, MSc, MS, \\ Stewart C. Wang, MD, PhD, and Matthew M. Davis, MD, MAPP
}

\begin{abstract}
Objectives: A mass casualty incident (MCI) may strain a health care system beyond surge capacity, affecting patterns of care for casualties and other patients. Prior studies of MCIs have assessed clinical care for casualty patients, but have not examined outcomes or expenditures for noncasualty inpatients in the same time period.

Methods: This was a retrospective analysis of administrative hospital claims in a state where an MCI with over 200 casualties occurred; two hospitals that admitted casualties of $>5 \%$ of their inpatient capacity were studied. The "surge period" was defined as 7 days after the MCI. Using diagnostic codes, patients admitted on the MCI day with diagnoses of burns or inhalation injury were included in the "MCI surge cohort." Patients admitted within a time frame of 7 days prior to 7 days after the MCI who were inpatients during the surge period were included in the "non-MCI surge cohort." The authors compared the MCI and non-MCI surge cohorts to a mutually exclusive reference cohort (all inpatients during 6 weeks prior to the MCI), regarding key outcomes of hospital length of stay (LOS) and hospital charges adjusted for age, sex, race/ethnicity, and severity of illness.

Results: Fifty-five patients met criteria for the MCI surge cohort, 1,369 for the non-MCI surge cohort, and 5,980 for the reference group. Compared with the reference group and adjusted for covariates, the mean $( \pm$ SD) hospital LOS was $4.90( \pm 1.85)$ days longer for the MCI surge cohort $(95 \%$ confidence interval $[C I]=1.67$ to 8.84$)$ and $1.34( \pm 0.16)$ days longer for the non-MCI surge cohort $(95 \% \mathrm{CI}=1.00$ to 1.65$)$. The MCI cohort also had significantly longer mean hospital LOS than the non-MCI surge cohort (difference $=3.56$ days; $95 \% \mathrm{CI}=0.36$ to 7.36 ). Also adjusted for covariates, mean $( \pm \mathrm{SD})$ total hospital charges for the MCI surge cohort were $\$ 22,349( \pm \$ 8,342)$ greater than for the reference group $(95 \% \mathrm{CI}=\$ 8,182$ to $\$ 39,485)$. Mean $( \pm S D)$ charges for the non-MCI surge cohort were $\$ 4,028( \pm \$ 633)$ greater than for the reference group $(95 \% \mathrm{CI}=\$ 2,792$ to $\$ 5,196)$. The MCI cohort also had higher mean total charges than the non-MCI surge cohort (difference $=\$ 18,321 ; 95 \% \mathrm{CI}=\$ 4,488$ to $\$ 34,980$ ).
\end{abstract}

Conclusions: When adjusted for severity of illness, casualty patients and noncasualty patients receiving concurrent hospital care have significantly longer LOS and higher charges than typical hospital patients at times unaffected by MCIs. Spillover effects from MCIs for noncasualty patients have not been previously described and have implications for clinical and hospital management in MCI and other high-surge circumstances.

ACADEMIC EMERGENCY MEDICINE 2012; 19:280-286 @ 2012 by the Society for Academic Emergency Medicine

From the Robert Wood Johnson Clinical Scholars Program (MA, HC, CRC, MMD), the Department of Emergency Medicine (MA), the Division of Pulmonary and Critical Care Medicine (CRC), the Department of Surgery (SCW), the Division of General Pediatrics and General Medicine (MMD), and the Gerald R. Ford School of Public Policy (MMD), University of Michigan, Ann Arbor, MI; and the Veterans Affairs Medical Center (MA), Ann Arbor, MI. Dr. Abir is currently with the George Washington University, Washington, DC.

Received June 15, 2011; revisions received June 19 and August 24, 2011; accepted September 16, 2011.

This work was supported by the Department of Veterans Affairs and the Robert Wood Johnson Foundation Clinical Scholars Program.

The authors have no relevant financial information or potential conflicts of interest to disclose.

Supervising Editor: Sandy Bogucki, MD, PhD.

A related commentary appears on page 318.

Address for correspondence and reprints: Mahshid Abir, MD, MSC; e-mail: mabir@mfa.gwu.edu. 
$\mathrm{P}$ rior research and expert initiatives regarding preparedness and response to mass casualty incidents (MCIs) and disasters have focused chiefly on outcomes for patients directly involved in the events. ${ }^{1-4}$ Multiple studies have investigated outcomes, such as time to definitive care, hospital length of stay (LOS), and mortality for casualties presenting from MCIs, ${ }^{1}$ and the effect of casualty load on these parameters, ${ }^{5-7}$ demonstrating conflicting results in some instances. A recent study by Peleg et al. ${ }^{8}$ found increased mortality in patients presenting from MCIs when compared to patients with similar injuries who did not present from a MCI. In contrast, outcomes for patients who are hospitalized for conditions unrelated to a MCI in facilities that have increased patient load in response to such incidents remain uninvestigated. How hospitals surge and what happens to patients during surge has been identified as a critical gap in understanding, especially when many hospitals frequently operate at or near inpatient capacity. ${ }^{9-11}$

Published literature related to hospital surge is mixed regarding implications for patient outcomes. A number of studies indicate that lower nurse-to-patient ratios are correlated with higher patient mortality, ${ }^{12-14}$ and a study of over 40 hospitals found that high hospital occupancy and low nurse staffing levels were found to be independently associated with higher in-hospital mortality. ${ }^{15}$ On the other hand, a study examining the effect of a California law enacted in 1999 requiring minimum staff-to-patient ratios did not show a significant effect on patient mortality. ${ }^{16}$ None of the aforementioned studies in the United States explicitly examined surge circumstances related to an MCI or disaster. A separate study of patient outcomes in German acute care hospitals during an "unexpected" surge in demand for resources (not in the context of an MCI) found longer hospital LOS, but no effect on emergency readmission or mortality for inpatients during the period of excess demand. ${ }^{17}$

To our knowledge, no studies have investigated the "spillover" effects of MCI-related hospital surge for noncasualty inpatients. Studying how noncasualty patient outcomes are associated with hospital surge in health systems responding to MCIs and disasters is an essential step in assessing the strengths and vulnerabilities of current hospital surge plans. Our study examines the effects of a specific MCI on hospitals and their patients, as measured by hospital LOS and total inpatient charges incurred.

\section{METHODS}

\section{Study Design}

This is a retrospective cohort study of a prospectively collected data set. We performed a retrospective analysis of the State Inpatient Dataset (SID) ${ }^{18}$ for a state in which an MCI resulting in over 200 casualties occurred, including dozens of burn victims. The SID is part of the Healthcare Cost and Utilization Project (HCUP), sponsored by the Agency for Healthcare Research and Quality (AHRQ). The SID is an all-payer inpatient care database containing demographic and clinical data, encompassing about $90 \%$ of all hospital discharges in

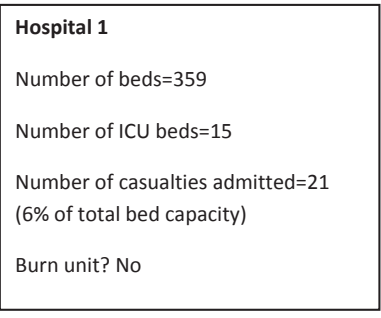

Hospital 1
Number of beds $=359$
Number of ICU beds $=15$
Number of casualties admitted $=21$
(6\% of total bed capacity)
Burn unit? No

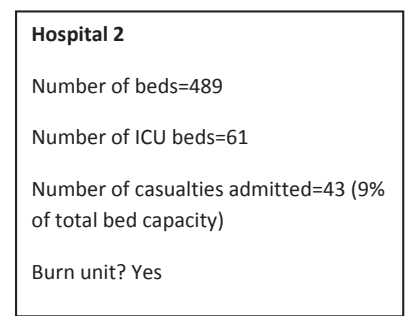

Figure 1. Characteristics of two hospitals to which mass casualty patients were admitted. ICU = intensive care unit.

the United States. This study was approved by the institutional review board (IRB) of the involved state's Department of Health and, as a study of deidentified patient data, was deemed not regulated by the IRB at our institution.

\section{Study Setting and Population}

Inclusion Criteria and Definitions. All of the state's hospitals received casualties from the MCI. To assure adequate analytic power, patients in the two hospitals that admitted the greatest number of casualties as a percentage of their baseline bed capacity ( $>5 \%$; Figure 1) were included in our analysis. The other hospitals in the state admitted an average of two casualties each, and therefore would not be expected to have experienced a significant surge in demand for resources.

Using discharge diagnosis codes (International Classification of Diseases, Version 9 [ICD-9]), all patients admitted on the calendar day of the MCI with a discharge diagnosis of burns or inhalation injury were identified as the "MCI surge cohort." Lacking a literature basis for defining a surge period, for the base-case analysis the authors defined the "surge period" as the seven days after the MCI (day of MCI + 7 days) based on common patterns of clinical care for patients with burns.

As comparison groups for the MCI surge cohort, we identified two other distinct cohorts. All patients admitted at any time within the 7 days prior or 7 days after the MCI and who remained inpatients for at least a portion of the surge period were included in the "non-MCI surge cohort." In an attempt to control for seasonal effects that other authors have found associated with inpatient mortality, ${ }^{15}$ the reference group was selected to comprise patients who were hospitalized in the same season as the MCI and non-MCI surge cohorts. Thus, our reference group included all patients admitted during the 6 weeks before the MCI and discharged prior to the MCI. The reference group and non-MCI surge cohort are mutually exclusive groups.

Study Variables. The SID contains more than 100 clinical and 100 nonclinical variables. Variables used in this study include admission month, admission year, age, race, sex, principal and secondary discharge diagnosis codes (ICD-9), hospital LOS, total hospital charges, hospital identification number, and disposition. Admission dates were acquired directly from the Department of Health of the involved state. Comorbidity was assessed from each patient's discharge diagnosis codes (ICD-9) 
using the Deyo modification of the Charlson score, a commonly used comorbidity index that is predictive of hospital mortality. ${ }^{19,20}$

\section{Data Analysis}

We used multiple linear regression to compare the MCI and the non-MCI surge cohorts to the reference group, with respect to hospital LOS and hospital charges incurred. To ensure robust regression modeling, we conducted a power analysis for each of the regressions and confirmed that the number of subjects in all our multivariate analyses exceeds the number of subjects required to attain a power $=0.9$ at alpha level $=0.05$. Given typical skew in the distributions of LOS and charges, both measures were log-transformed for this analysis. A separate regression was performed for each outcome, and each regression model included the potential confounding variables age, sex, race/ethnicity, and comorbidity (using the Charlson Comorbidity Index). ${ }^{19,20}$ No variable selection was performed; rather, variables identified at the outset of the study as conceptually important were retained in the final analyses. We repeated these analyses after including hospital as a fixed effect in the model. When adjusting for unobservable hospital-level effects (i.e., fixed effects), we included hospital as a dichotomous variable in the regression model. This approach accounts for correlation in the outcomes of patients cared for in the same hospital. Regression coefficients were used to generate predicted values of the LOS and charge outcomes. To back-transform log-transformed results, we used a nonparametric retransformation method (smearing estimate). ${ }^{21}$ Using this method, the expected values for untransformed outcomes (i.e., total charges and hospital LOS) were obtained by using the sample mean for residuals, rather than the direct standard error from the normal distribution of the coefficients. All analyses were conducted using Stata version 11 (StataCorp, College Station, TX). All tests were two-tailed, and a $\mathrm{p}$-value of $<0.05$ was considered statistically significant for single and multiple comparisons. We did not account for multiple comparisons in our calculation of p-values.

Regression diagnostics for both total charges and hospital LOS were conducted. The assumption for the absence of strongly influential outliers is met in regressions for both outcomes. The assumption for homoscedasticity is met in the regression of total hospital charges, but not in the regression of total hospital LOS. Therefore, robust standard errors are obtained for the regression of hospital LOS and for the respective p-values. Our main explanatory variable of interest is a categorical variable (reference vs. MCI and non$\mathrm{MCI}$ ); therefore, linearity is not assumed in estimating the effect on MCI and non-MCI cohorts.

Sensitivity Analyses. Given uncertainty in some of the parameters of the analyses, we conducted sensitivity analyses. First, we varied the surge period definition, as short as 3 days and as long as 14 days, to assess whether the findings were robust to alternative definitions of the surge period. Next, we assessed whether findings were sensitive to alternative reference periods in the year of the MCI. Finally, we conducted an analysis stratified by hospital, to examine explicitly the extent to which spillover effects may differ by institution. For this purpose, separate multiple linear regressions were conducted for each of the two hospitals comparing MCI and non-MCI surge cohorts to hospital-specific reference groups while adjusting for age, sex, race/ ethnicity, and severity of illness. Skewed, dependent variables were back-transformed, as in the primary analysis, using smearing estimates.

\section{RESULTS}

\section{Study Population}

Fifty-five patients met inclusion criteria for the MCI surge cohort, 1,369 for the non-MCI surge cohort, and 5,980 for the reference group. This MCI primarily involved young, previously healthy males, reflected in demographic and clinical characteristics of the MCI cohort (Table 1). As expected, there were no significant differences in the demographic characteristics of the non-MCI surge cohort and the reference group.

We compared inpatient mortality between the MCI and non-MIC surge cohorts and the reference group, and no significant differences were observed. A separate analysis comparing inpatient mortality between these cohorts and the reference group for patients

Table 1

Demographics of the Study Cohorts

\begin{tabular}{|c|c|c|c|c|c|c|}
\hline $\begin{array}{l}\text { Patient } \\
\text { Characteristic }\end{array}$ & $\begin{array}{l}\text { MCl Surge } \\
\text { Cohort* } \\
(n=55)\end{array}$ & $\begin{array}{c}\text { Non-MCl Surge } \\
\text { Cohort } \dagger \\
(n=1,369)\end{array}$ & $\begin{array}{l}\text { Reference } \\
\text { Patients } \\
(n=5,980)\end{array}$ & $\begin{array}{c}\text { p-valuesł (MCl } \\
\text { Surge vs. } \\
\text { Reference) }\end{array}$ & $\begin{array}{l}\text { p-valuest } \\
\text { (Non-MCl } \\
\text { Surge vs. } \\
\text { Reference) }\end{array}$ & $\begin{array}{c}\text { p-values: } \\
\text { ( } \mathrm{MCl} \text { vs. } \\
\text { Non- } \mathrm{MCl} \text { Surge) }\end{array}$ \\
\hline Mean (SD) age, yr & $35(13)$ & 52 (27) & $50(28)$ & $<0.001$ & 0.20 & 0.007 \\
\hline Sex (\%) male & 64 & 46 & & 0.03 & 0.08 & 0.11 \\
\hline Race (\%) white & 93 & 86 & 84 & 0.09 & 0.08 & 0.06 \\
\hline $\begin{array}{l}\text { Mean Charlson } \\
\text { score (SD) }\end{array}$ & $0.34(1.19)$ & $1.17(1.62)$ & $1.06(1.56)$ & 0.001 & 0.02 & 0.04 \\
\hline \multicolumn{7}{|c|}{$\begin{array}{l}\mathrm{MCl}=\text { mass casualty incident. } \\
\text { *The } \mathrm{MCl} \text { surge cohort includes all patients admitted on the day of the } \mathrm{MCl} \text { with burns or inhalation injury. } \\
\dagger \text { The non- } \mathrm{MCl} \text { surge cohort includes all patients admitted during the } 7 \text { days prior or } 7 \text { days after the } \mathrm{MCl} \text { who were inpatient } \\
\text { during the 7-day surge period. } \\
\text { tp-values for age were obtained from multiple regression and logistic regression. }\end{array}$} \\
\hline
\end{tabular}




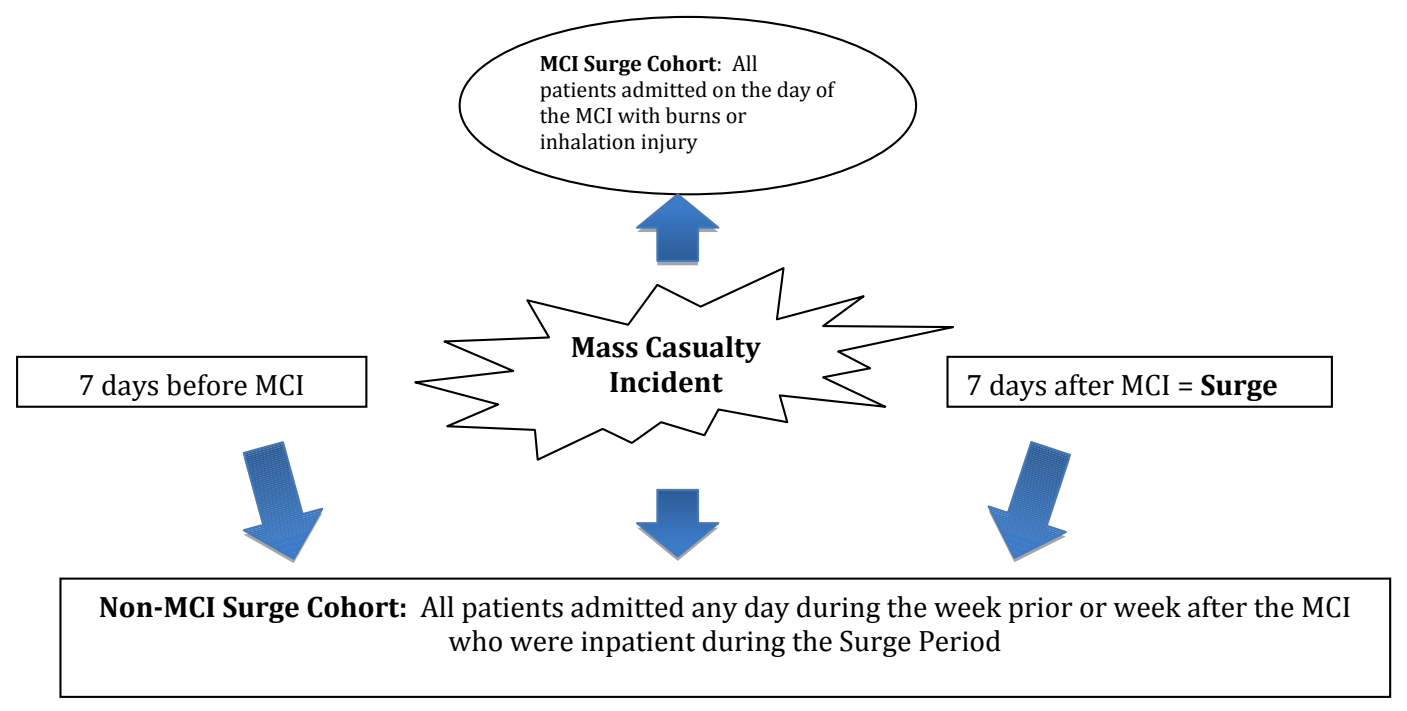

Figure 2. Definitions and timeline for three mutually exclusive $\mathrm{MCl}$ study cohorts. $\mathrm{MCl}=$ mass casualty incident.

admitted to the intensive care unit (ICU) or the cardiac care unit also did not reveal a significant difference. These data are not reported due to the concern for not having adequate power to detect a rare event such as mortality.

Differences in LOS and Hospital Charge, by Cohort Our unadjusted results indicate a longer hospital LOS for both the MCI (mean $\pm \mathrm{SD}=10.01 \pm 1.80$ days; $95 \%$ confidence interval $[\mathrm{CI}]=6.87$ to 13.79 days) and the non-MCI (mean $\pm \mathrm{sd}=6.38 \pm 0.14$ days; $95 \%$ CI $=6.08$ to 6.67 days) surge cohorts, when compared to the reference group (mean $\pm \mathrm{SD}=5.09 \pm 0.06$ days; $95 \%$ $\mathrm{CI}=4.97$ to 5.20 days). Unadjusted results for total hospital charges incurred also indicate greater charges for both the MCI (mean $\pm \mathrm{sd}=\$ 40,353 \pm \$ 8,093 \quad 95 \%$ $\mathrm{CI}=\$ 27,267$ to $\$ 58,263$ ) and non-MCI (mean $\pm \mathrm{SD}=$ $\$ 21,608 \pm \$ 561 ; 95 \% \quad C I=\$ 21,819$ to $\$ 23,982)$ surge cohorts, when compared to the reference group (mean $\pm \mathrm{sd}=\$ 17,796 \pm \$ 247 ; \quad 95 \%=\mathrm{CI} \quad \$ 18,405$ to $\$ 19,361)$.

Adjusted for patient age, sex, race/ethnicity, and comorbidities, LOS and hospital charges are compared for the two study cohorts in Figure 2 relative to the reference group. The mean $( \pm$ SD) hospital LOS was $4.90( \pm 1.85)$ days longer for the MCI surge cohort compared to the reference group $(95 \% \quad \mathrm{CI}=1.67$ to
8.84 days; $p<0.001)$. The mean $( \pm S D)$ LOS for the nonMCI surge cohort was $1.34( \pm 0.16)$ days longer than the reference group ( $95 \% \mathrm{CI}=1.00$ to 1.65 days; $\mathrm{p}<0.001$ ). When comparing the MCI to the non-MCI surge cohort, the MCI cohort had significantly longer mean hospital LOS (difference $=3.56$ days; $95 \% \mathrm{CI}=0.36$ to 7.36 days; $\mathrm{p}<0.001)$.

Also adjusted for covariates, the mean $( \pm \mathrm{SD})$ total hospital charges for the MCI surge cohort were $\$ 22,349( \pm \$ 8,342)$ higher than for the reference group $(95 \% \mathrm{CI}=\$ 8,182$ to $\$ 39,485 ; \mathrm{p}<0.001)$. The mean $( \pm \mathrm{SD})$ charges for the non-MCI surge cohort were $\$ 4,028( \pm \$ 633)$ higher than the total charges associated with the reference group $(95 \% \mathrm{CI}=\$ 2,792$ to $\$ 5,196$; $\mathrm{p}<0.001)$. When comparing the MCI to the non-MCI surge cohort, we found that the MCI cohort had higher mean total charges (difference $=\$ 18,321$; $95 \%$ $\mathrm{CI}=\$ 4,488$ to $\$ 34,980 ; \mathrm{p}<0.001)$. All reported regression model means for hospital LOS and total charges represent back-transformed values.

\section{Sensitivity Analyses}

Adjusting for fixed rather than random hospital effects did not alter the results of our analysis (data available from the authors upon request). The results of the stratified analyses examining each of the two hospitals independently are presented in Table 2. In Hospital 1, there

Table 2

Hospital LOS and Total Charges, for MCl and Non-MCI Surge Cohorts Versus Reference Patients at Each Hospital

\begin{tabular}{|c|c|c|c|c|c|c|c|}
\hline \multirow[b]{2}{*}{ Parameter } & \multirow[b]{2}{*}{ Comparison Groups } & \multicolumn{3}{|c|}{ Hospital 1} & \multicolumn{3}{|c|}{ Hospital 2} \\
\hline & & Coefficient & $\mathrm{p}$-value & $95 \% \mathrm{Cl}$ & Coefficient & $\mathrm{p}$-value & $95 \% \mathrm{Cl}$ \\
\hline \multirow[t]{2}{*}{ Hospital LOS } & $\mathrm{MCl}$ vs. reference & -1.44 & 0.15 & -3.39 to 1.16 & 1.10 & $<0.001$ & 3.78 to 15.46 \\
\hline & Non-MCl vs. reference & 2.41 & 0.02 & 0.72 to 1.91 & 0.17 & $<0.001$ & 0.95 to 1.7 \\
\hline \multirow[t]{2}{*}{ Total charges } & $\mathrm{MCl}$ vs. reference & 0.19 & 0.29 & $-\$ 6,626$ to $\$ 6,582$ & 1.24 & $<0.001$ & $\$ 17,129$ to $\$ 74,977$ \\
\hline & Non-MCl vs. reference & 0.09 & 0.02 & $\$ 1,599$ to $\$ 4,386$ & 0.19 & $<0.001$ & $\$ 3,349$ to $\$ 7,070$ \\
\hline
\end{tabular}


were no significant differences in hospital LOS or hospital charges when comparing the MCI surge cohort to the reference group. In contrast, for the nonMCI surge cohort versus the reference group there were differences in mean hospital LOS (difference $=1.28 ; 95 \% \mathrm{CI}=0.72$ to $1.91 ; \mathrm{p}=0.02$ ) and mean total charges (difference $=\$ 2,970 ; 95 \% \mathrm{CI}=\$ 1,599$ to $\$ 4,386 ; p=0.02)$. At Hospital 2, mean LOS was longer for the MCI surge cohort than for the reference group (difference $=9.28 ; 95 \% \mathrm{CI}=3.78$ to $15.46 ; \mathrm{p}<0.001$ ), and mean total hospital charges were also higher (difference $=\$ 42,273 ; 95 \% \quad C I=\$ 17,129$ to $\$ 74,976 ; p<$ 0.001). In addition, the non-MCI surge cohort had longer mean hospital LOS (difference $=1.32$; $95 \%$ $\mathrm{CI}=0.95$ to $1.70 ; \mathrm{p}<0.001)$ and higher mean hospital charges (difference $=\$ 5,244 ; 95 \% \mathrm{CI}=\$ 3,349$ to $\$ 7,070$; $\mathrm{p}<0.001)$ compared to the reference group.

Our findings were robust to the duration of the surge period (Figure 1 and Data Supplement S1, available as supporting information in the online version of this paper) and to selection of alternate months of the year as reference periods (Figure 3 and Data Supplement S1), presented as ordinary least squares coefficients.
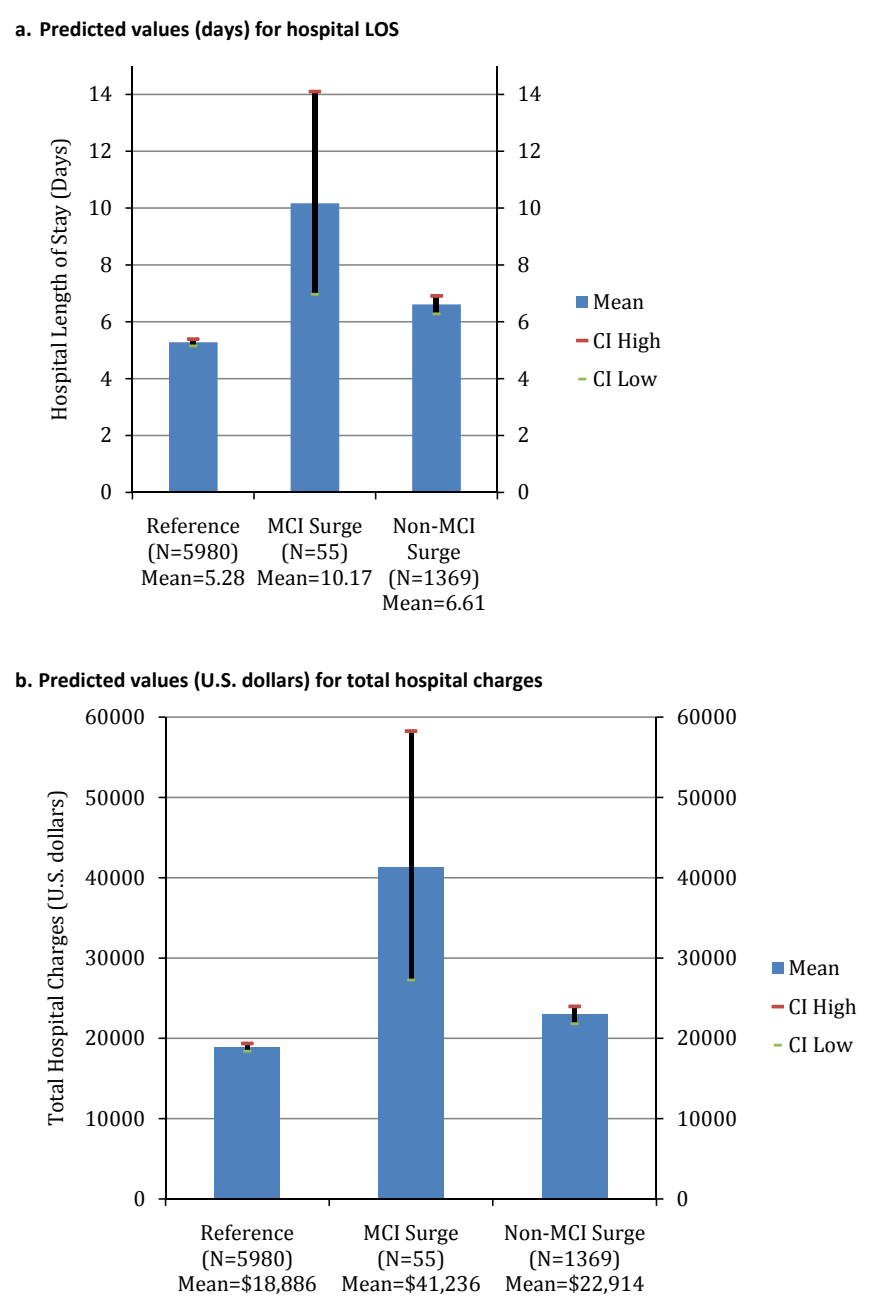

Figure 3. Predicted values for comparisons between $\mathrm{MCl}$ and non-MCl surge cohorts with the reference group with respect to hospital LOS (a) and total hospital charges (b).
We found that hospital LOS for the MCI and non-MCI surge cohorts were consistently higher than all other reference periods. Hospital charges for the MCI surge cohort were consistently higher versus all other reference periods. In contrast, hospital charges for the non-MCI surge cohort were similar to reference periods in October, November, and December.

\section{DISCUSSION}

Disaster experts have recognized a paucity of research regarding the effectiveness of interventions and associated outcomes during periods of hospital surge. ${ }^{22}$ Studying patient outcomes related to specific MCIs or disasters may help evaluate the effectiveness of current hospital surge plans. Our findings indicate that both hospital LOS and total hospital charges are significantly higher in the MCI surge cohort when compared to the reference group. Given that the MCI surge cohort is primarily composed of burn patients, who typically have long hospitalizations and large hospital charges, ${ }^{23}$ we were not surprised to find that the MCI surge cohort had significantly greater resource utilization than typical patients at these hospitals.

We also found a statistically significant difference in the hospital LOS and total charges for the non-MCI surge cohort versus reference patients-i.e., a "spillover" effect of the MCI on the measures of care received by patients not involved in the MCI. These outcomes may represent inefficiencies in the system as a result of an increase in demand for personnel, space, and material resources due to the surge in admissions from the MCI.

Our stratified analysis, examining each of the two hospitals under study, illuminated key similarities and distinctions in the responses of the two hospitals to the MCI. At both hospitals, the non-MCI surge cohort experienced longer LOS and higher charges compared with the reference group, illustrating how perturbations of the institution's usual care routines (e.g., lower nurse-to-patient staffing ratios or utilization of critical care and ward beds by teams other than the usual) in the setting of surge can lead to less efficient care.

On the other hand, we found differences in resource utilization related to the MCI cohort itself for Hospital 1 compared with Hospital 2. At Hospital 1, without a dedicated burn unit, we did not find significant differences in either hospital LOS or total charges among the patients in the MCI surge cohort relative to the reference group. Based on the available literature reporting details of this MCI, Hospital 1 transferred 18 burn patients and admitted 21 patients of the total of 82 patients that it treated. Our null findings regarding the MCI cohort suggest that patients with more severe burns were transferred out to other institutions with readily available burn specialty services. In contrast, staff at Hospital 2 (with a dedicated burn unit) treated 68 patients, admitting 43 and transferring eight patients to other institutions. At this hospital, LOS and total charges incurred were increased for the MCI surge cohort relative to the reference group, consistent with our hypothesis about effects of surge. Therefore, care patterns for the MCI surge cohorts appear related to 
the number and severity of casualty patients, whereas patterns for the non-MCI surge cohorts may be more consistently affected by the shock of MCI care efforts.

We did not find a significant difference in mortality when comparing the MCI and non-MCI surge cohorts to the reference group. In other words, the hospitals appeared to perform as well as their usual benchmarks in the challenging setting of an MCI. However, we are aware that these cohorts and the low frequency of mortality limit the robustness of our estimates of this outcome; this is another rationale for looking at measures such as LOS and charges as well, to examine how hospitals perform in response to MCIs.

\section{LIMITATIONS}

This study has a number of limitations. This is an analysis of outcomes related to the response of two hospitals to a predominantly burn MCI, and thus the results may not be generalizable to other hospitals and may not apply to hospital surge in response to other types (and sizes) of MCIs that may strain health systems to a lesser or greater extent. The unique characteristics of burn care (time- and resource-intensive) may not be applicable to other types of MCIs.

Another limitation is that this study does not definitively identify the causes of the differences in LOS and charges among the cohorts. This limitation is related to the fact that our analysis was based exclusively on administrative claims data, which do not shed much light on logistic responses of hospitals or changes to usual patterns of care. We hasten to add that this limitation of our study also reflects the current state of research regarding MCIs in the United States, which is underdeveloped relative to many other research domains regarding health services.

A third limitation of our work is that the SID includes encounter-level data but not patient-linked data. For this reason, we were not able to examine another outcome of interest in the management of MCIs-namely, whether rates of readmission change for patients treated in the surge period. Readmission rates were included in a recent analysis from Germany and found not to differ related to unexpected surges in patient flow. ${ }^{17}$ However, the twin challenges of discharging potentially eligible inpatients at the time the MCI occurs, and managing immediately scarce resources with scarce staff during the surge period, make readmission theoretically more likely, and this measure would optimally be part of future studies. Finally, we performed multiple statistical tests, but did not adjust for multiple comparisons. Readers should interpret the significance of results near our significance-threshold $(p<0.05)$ with caution.

This study is an attempt at understanding the effects of a specific MCI on a health system as measured by hospital LOS and total hospital charges incurred. A more extensive study of the response of these two hospitals to this MCI will be required to further our understanding of the factors that have contributed to the increases in these parameters for non-MCI inpatients during the surge period. Such information will be important in evaluating the strengths and limitations of current hospital surge plans. Similar analyses of hospital surge in response to other burn MCIs will be necessary to assess whether similar results are observed. Studying hospital surge in response to burn MCIs of different sizes will allow an estimation of maximum burn surge capacity at given institutions. Moreover, studying MCIs with different types of predominant injuries (e.g., trauma, chemical exposure) will permit broader appreciation of whether different MCI forms stress hospital systems similarly or differently.

\section{CONCLUSIONS}

We have identified statistically significant increases in hospital length of stay and total charges for non-mass casualty incident patients at two hospitals that admitted the greatest number of casualties from a specific mass casualty incident. Further studies are required to determine the mechanisms through which these increases represent a spillover effect, as a result of strains and inefficiencies in the system during a period of patient surge. With better understanding of surge response, institutions may improve their surge plans in order to minimize effects on non-mass casualty incident patients, while optimizing care for mass casualty incident patients.

The authors are grateful to the Robert Wood Johnson Foundation Clinical Scholars program for the support of Drs. Abir, Cooke, and Davis. We also like to acknowledge Acham Gebremariam for his statistical support. The findings and views expressed herein are those of the authors and not of their institutions or funders.

\section{References}

1. Ball CG, Kirkpatrick AW, Mulloy RH, Gmora S, Findlay C, Hameed M. The impact of multiple casualty incidents on clinical outcomes. J Trauma. 2006; 61:1036-9.

2. Mackie DP, Koning HM. Fate of mass burn casualties: implications for disaster planning. Burns. 1990; 16:203-6.

3. Edgar DW, Wood F, Goodwin-Walters A. First response, rehabilitation, and outcomes of hand and upper limb function: survivors of the Bali bombing disaster. A case series report. J Hand Ther. 2006; 19:283-98.

4. Peleg K, Aharonson-Daniel L, Stein M, et al. Gunshot and explosion injuries: characteristics, outcomes, and implications for care of terror-related injuries in Israel. Ann Surg. 2004; 239:311-8.

5. Shoher A, Chang DC, Efron D, Cornwell EE III, Haut ER. Multiple, simultaneous trauma patients: are they worse off? J Trauma. 2006; 61:611-5.

6. Hirshberg A, Scott BG, Granchi T, Wall MJ Jr, Mattox KL, Stein M. How does casualty load affect trauma care in urban bombing incidents? A quantitative analysis. J Trauma. 2005; 58:686-95.

7. Hirshberg A, Holcomb JB, Mattox KL. Hospital trauma care in multiple-casualty incidents: a critical view. Ann Emerg Med. 2001; 37:647-52.

8. Peleg K, Rozenfeld M, Stein M. Poorer outcomes for mass casualty events victims: is it evidence based? J Trauma. 2010; 69:653-9. 
9. Agency for Healthcare Research and Quality. Bioterrorism Preparedness Research Portfolio, 2002, 2003. Available at: http://www.ahrq.gov/research/ bioterport.htm\#Surge. Accessed May 5, 2011.

10. Agency for Healthcare Research and Quality. Bioterrorism Preparedness Research Portfolio, 2004, 2005. Available at: http://www.ahrq.gov/research/ btport05.htm. Accessed May 5, 2011.

11. Phillips S. Current status of surge research. Acad Emerg Med. 2006; 13:1103-8.

12. Aiken L, Clarke S, Sloane D, Sochalski J, Silber J. Hospital nurse staffing and patient mortality, nurse burnout, and job dissatisfaction. JAMA. 2002; 288:1987-93.

13. Needleman J, Buerhaus P, Pankratz VS, Leibson CL, Stevens SR, Harris M. Nurse staffing and inpatient hospital mortality. NEJM. 2011; 364:1037-45.

14. Sasichay-Akkadechanunt T, Scalzi CC, Jawad AF. The relationship between nurse staffing and patient outcomes. J Nurs Adm. 2003; 9:478-85.

15. Schilling PL, Campbell DA, Englesbe MJ, Davis MM. A comparison of in-hospital mortality risk conferred by high hospital occupancy, differences in nurse staffing levels, weekend admission, and seasonal influenza. Med Care. 2010; 48:224-32.

16. Evans WN, Kim B. Patient outcomes when hospitals experience a surge in admissions. J Health Econ 2006; 25:365-88.

17. Schwierz C, Augurzky B, Focke A, Wasem J. Demand, selection and patient outcomes in German acute care hospitals. J Health Econ 2012; 21: 209-21.

18. HCUP State Inpatient Databases (SID). Healthcare Cost and Utilization Project (HCUP). 2005-2009. Agency for Healthcare Research and Quality,
Rockville, MD. Available at: http://www.hcup-us.ahrq. gov/sidoverview.jsp. Accessed Nov 8, 2011.

19. Charlson ME, Pompei $P$, Ales KL, MacKenzie CR. A new method of classifying prognostic comorbidity in longitudinal studies: development and validation. J Chronic Dis. 1987; 40:373-83.

20. Deyo RA, Cherkin DC, Ciol MA. Adapting a clinical comorbidity index for use with ICD-9-CM administrative databases. J Clin Epidemiol. 1992; 45:613-9.

21. Duan N. Smearing estimates: a nonparametric retransformation method. J Am Stat Assoc. 1983; 78:605-10.

22. Barbisch D, Haik J, Tessone A, Hanfling D. Surge Capacity. In: Koenig KL, Schultz CH (eds). Disaster Medicine: Comprehensive Principles and Practices. New York, NY: Cambridge University Press, 2010, pp 33-50.

23. Barillo DJ. Planning for burn mass casualty incidents. J Trauma. 2007; 62(6 Suppl):S68.

\section{Supporting Information}

The following supporting information is available in the online version of this paper:

Data Supplement S1. Containing Figures 1 and 2 depicting results of sensitivity analyses using alternate definitions of the surge period and alternate months of the year as the reference group.

The document is in PDF format.

Please note: Wiley Periodicals Inc. is not responsible for the content or functionality of any supporting information supplied by the authors. Any queries (other than missing material) should be directed to the corresponding author for the article. 